\title{
The anabolic skeletal muscle response to acute resistance exercise is not impaired in rats fed a ketogenic diet
}

\author{
C Brooks Mobley ${ }^{1 *}$, Angelia M Holland ${ }^{1}$, Wesley C Kephart ${ }^{1}$, Ryan P Lowery ${ }^{2}$, Petey W Mumford ${ }^{1}$, \\ Anna E McCloskey ${ }^{1}$, Joshua J Shake ${ }^{1}$, Paulo Mesquita ${ }^{1}$, Jacob M Wilson ${ }^{2}$, Michael D Roberts ${ }^{1,3}$ \\ From The Twelfth International Society of Sports Nutrition (ISSN) Conference and Expo \\ Austin, TX, USA. 11-13 June 2015
}

\section{Background}

Many individuals that resistance train consume a typical Western diet (WD) comprised of protein, carbohydrates (many of which are sugar), and fat. Recent enthusiasm has surrounded the use of a ketogenic diet for weight loss and muscle sparing, although it is uncertain as to whether low carbohydrate diets can optimize the anabolic response to resistance training.

\section{Methods}

This study examined the effects of KD versus WD on the anabolic response to resistance exercise using a rodent leg-kicking resistance exercise model. Male Sprague-Dawley rats ( 9-10 weeks of age) were provided isocaloric amounts of either a KD $(5.2 \mathrm{kcal} / \mathrm{g}, 20.2 \%$ protein, $10.3 \%$ carbohydrate, $69.5 \%$ fat; $\mathrm{n}=30$ ) or WD (4.5 kcal/g, $15.2 \%$ protein, $42.7 \%$ carbohydrate, $42.0 \%$ fat; $n=32$ ) for 6 weeks. During week 7 , the right-leg plantarflexor muscles of each rat were acutely exercised under isoflurane anesthesia using high-frequency electrical stimulations (4 sets of 8 repetitions with 2 min recovery between sets). Rats were then sacrificed at $90 \min (\mathrm{n}=8$ per group), $180 \min (\mathrm{n}=8$ per group), or $270 \mathrm{~min}(\mathrm{n}=8$ per group) following exercise and intraperitoneal puromycin injections were provided $30 \mathrm{~min}$ prior to each sacrifices as a tracer for muscle protein synthesis (MPS). A subset of unexercised limbs from WD $(\mathrm{n}=8)$ and KD $(\mathrm{n}=8)$ were used as a nonexercise (non-EX) control comparison.

\section{Results}

There was a main time effect for MPS, as it was significantly greater at 90, 180 and $270 \mathrm{~min}$ in both groups versus the non-EX condition ( $\mathrm{p}<0.001)$, although there was no between group effect $(\mathrm{p}=0.59)$ or group"time interaction $(\mathrm{p}=0.87)$. There was a main time effect for phosphorylated (p)-4E-BP1 (Thr37/46), as it was significantly greater at 90, 180 and $270 \mathrm{~min}$ in both groups versus the non-EX condition $(\mathrm{p}=0.001)$, although there was no between group effect $(\mathrm{p}=0.85)$ or group"time interaction $(\mathrm{p}=0.93)$. There was a main time effect for $\mathrm{p}$-rps6 (Ser235/236), as it was significantly greater at 90,180 and $270 \mathrm{~min}$ in both groups versus the non-EX condition $(\mathrm{p}=$ $0.002)$, although there was no between group effect ( $\mathrm{p}=$ $0.99)$ or group $\mathrm{p}^{*}$ time interaction $(\mathrm{p}=0.79)$. There was no time effect $(p=0.31)$, between group effect $(p=0.42)$ or group*time interaction $(\mathrm{p}=0.22)$ for $\mathrm{p}$-AMPK $\alpha($ Thr172).

\section{Conclusions}

These data demonstrate that rats fed a ketogenic diet present a similar anabolic response to resistance exercise compared to rats fed a Western diet.

\begin{abstract}
Authors' details
${ }^{1}$ School of Kinesiology, Auburn University, Auburn, AL, USA. ${ }^{2}$ Department of Health Sciences and Human Performance, The University of Tampa, Tampa, FL, USA. ${ }^{3}$ Edward Via College of Osteopathic Medicine - Auburn Campus, Auburn, AL, USA.
\end{abstract}

Published: 21 September 2015 